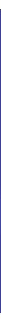

\title{
A UNIVERSIDADE PARA A INDÚSTRIA DO FUTURO
}

\author{
Mauricio de Campos Porath \\ Universidade Federal de Santa Catarina \\ mauricio.porath@ufsc.br \\ Xisto Lucas Travassos Júnior \\ Universidade Federal de Santa Catarina \\ lucas.travassos@ufsc.br \\ Jonas Tilp \\ TreeCom Coworking \\ jonas.tilp@treecom.club
}

Resumo

$\mathrm{Na}$ última década, a indústria mundial tem passado por rápidas e profundas mudanças induzidas pela introdução de novas tecnologias digitais. Essa mudança de paradigma é denominada quarta revolução industrial ou "Indústria 4.0". Os impactos dessas mudanças nas relações de trabalho e no sistema de educação são inevitáveis. O $1^{\circ}$ Workshop Internacional: A Universidade para a Indústria do Futuro - U4i 2018 objetivou promover o debate acerca da relação universidade-indústria frente à quarta revolução industrial. O U4i 2018 contou com participantes da universidade e de empresas. O evento incluiu uma atividade denominada World Café, na qual os participantes buscaram respostas a seis questões pertinentes ao tema do evento. Este artigo objetiva apresentar os resultados das discussões realizadas no âmbito dessa atividade. Os participantes destacaram a necessidade de se melhorar a comunicação e a integração e de se aumentar a agilidade nas relações universidade-indústria. Também ressaltou-se que os empresários precisam conscientizar-se de que devem assumir uma postura mais proativa e arrojada frente à nova realidade. Em relação à atuação governamental no contexto da cooperação universidade-indústria, criticou-se a falta de continuidade de programas de fomento.

Palavras-chave: Quarta Revolução Industrial. Indústria 4.0. Cooperação Universidade-indústria. World Café.

\section{THE UNIVERSITY FOR THE INDUSTRY OF THE FUTURE}

\begin{abstract}
In the last decade, global industry undergoes rapid and profound changes induced by the introduction of new digital technologies. This paradigm shift is called fourth industrial revolution or "Industry 4.0 ". The impacts of these changes on labor relations and the education systems are inevitable. The 1 st International Workshop: The University for the Industry of the Future - U4i 2018 aimed to promote the debate about university-industry relation within the context of the the fourth industrial revolution. U4i 2018 was attended by both university and business participants. The event included an activity called World Café, in which participants sought answers to six questions pertinent to the theme of the event. This paper presents the results of the discussions carried out under this activity. Participants emphasized the need to improve communication and integration and to increase agility in university-industry relations. It was also emphasized that entrepreneurs need to be aware that they must take a more proactive and bold approach to the new reality. In relation to the governmental action in the context of industry university cooperation, it was criticized the lack of continuity of development programs.
\end{abstract}

Keywords: Forth Industrial Revolution. Industry 4.0. University-industry Cooperation. World Café.

\section{LA UNIVERSIDAD PARA LA INDUSTRIA DEL FUTURO}

\section{Resumen}

La industria mundial pasa por rápidos y profundos cambios inducidos por la introducción de nuevas tecnologías digitales. Este cambio de paradigma se denomina cuarta revolución industrial o "Industria 4.0". Los impactos de estos cambios en las relaciones de trabajo y en el sistema educativo son inevitables. El 1 er Workshop Internacional: La Universidad para la Industria del Futuro - U4i 2018 objetivó promover el debate acerca de la relación universidad-industria frente a la cuarta revolución industrial. El U4i 2018 contó con participantes de la universidad y de empresas. El evento incluyó una actividad denominada World Café, en la cual los participantes buscaron respuestas a seis cuestiones pertinentes al tema del evento. Este artículo tiene como objetivo presentar los resultados de las discusiones mantenidas dentro de esta actividad. Los participantes destacaron la necesidad de mejorar la comunicación y la integración y de aumentar la agilidad en las relaciones universidad-industria. También se resalta que los empresarios necesitan concientizarse que deben asumir una postura más proactiva y arrojada frente a la nueva realidad. En relación a la actuación gubernamental en el contexto de la cooperación universidad-industria, se criticó la falta de continuidad de programas de fomento.

Palavras clave: Cuarta Revolución Industrial. Industria 4.0. Cooperación Universidad-industria. World Café. 


\section{INTRODUÇÃO}

A indústria mundial passa por rápidas e profundas mudanças. Essas mudanças estão principalmente relacionadas à intensa automatização de processos e ao emprego de ferramentas de tecnologia da informação em todo o ciclo de vida de produtos. A introdução de novas tecnologias digitais no ambiente industrial, como a internet das coisas, a inteligência artificial, a análise de grandes volumes de dados (big data) e a computação nas nuvens, tem potencial para estabelecer novos paradigmas, tanto no ambiente competitivo das empresas, quanto no mercado de trabalho. Convencionou-se denominar essas mudanças de paradigma de "quarta revolução industrial" ou "Indústria 4.0".

Essa nova realidade representa uma oportunidade para a prosperidade econômica e o desenvolvimento social. No entanto, para que essa oportunidade possa efetivamente ser aproveitada, é necessário que todos os envolvidos estejam abertos a mudanças, em especial nos sistemas de educação e nas relações de trabalho, conforme apontado por Klaus Schwab, fundador e presidente executivo do Fórum Econômico Mundial, no prefácio do estudo Future of Jobs 2018 (WORLD ECONOMIC FORUM, 2018).

Atenta a esse movimento, a Universidade Federal de Santa Catarina promoveu nos dias 31 de outubro e $1^{\circ}$ de novembro de 2018 o $1^{\circ}$ Workshop Internacional - A Universidade para a Indústria do Futuro - U4i 2018. O evento foi realizado no Centro Tecnológico de Joinville da UFSC e contou com a parceria da universidade alemã TH Ingolstadt e do Perini Business Park. O U4i 2018 reuniu professores, estudantes e pesquisadores da universidade com empresários, executivos e profissionais do meio industrial. Promoveu-se, assim, o tão necessário debate acerca da interação universidade-indústria diante das mencionadas transformações.

O U4i 2018 contou com a participação de 70 profissionais de empresas de diversos segmentos e de todos os portes, 30 professores/pesquisadores e 60 estudantes de graduação e pós-graduação. A programação do evento incluiu palestras, mesas redondas e visitas a laboratórios, além de uma atividade de integração e brainstorming denominada U4i World Café. Dessa última atividade resultou uma profícua discussão, na qual buscaram-se respostas a seis perguntas relacionadas ao tema do evento. Este artigo objetiva apresentar os resultados dessa discussão. 


\section{METODOLOGIA}

World Café é uma metodologia para incentivar a participação estimulando o diálogo e o estabelecimento de relações entre os participantes. No âmbito do U4i, o objetivo desta dinâmica era aprimorar a relação entre universidade e indústria. Desta forma, o método foi modificado para atender a essa especificidade.

As modificações no método consideraram: o contexto, número de participantes e objetivos do evento. Desta forma, o World Café do U4i teve as seguintes características:

1) Ambiente acolhedor: mesas preparadas para acomodar cinco participantes com assistência para anotações dos assuntos discutidos de forma acessível e rápida;

2) Apresentação e boas-vindas: Jonas Tilp, empreendedor e idealizador da Treecom Coworking (instalada no Perini Business Park), atuou como facilitador do evento, recebendo os convidados para o World Café e apresentando o contexto, os objetivos e orientando os participantes quanto os resultados pretendidos;

3) Rodadas: O processo de interação entre os participantes foi dividido em cinco rodadas de até dez minutos de conversas entre as pessoas em cada mesa. Cada mesa teve um "host", sendo este um docente da UFSC. Passados dez minutos, todos os participantes, exceto o host, mudaram de mesa conforme programação. Ao chegar à nova mesa, os participantes foram informados pelo host sobre o que foi discutido na rodada anterior;

4) Perguntas: em cada mesa propôs-se uma pergunta ligada diretamente ao objetivo da dinâmica, focada em discussões para que as metas do evento fossem atendidas;

5) Conclusão e encerramento: ao final das rodadas, o facilitador do evento fez uma plenária sobre os pontos discutidos e possíveis ações futuras;

6) Cada mesa pode registrar de forma livre os pontos discutidos. Estes apontamentos foram catalogados e disponibilizados para a comunidade como um todo.

O U4i World Café contou com a participação de oito representantes de universidades e 17 representantes de empresas de diversos portes e segmentos.

\section{RESULTADOS E ANÁLISES}

As perguntas formuladas aos participantes do U4i World Café foram:

- Como é a indústria do futuro?

- O que falta para que a indústria nacional seja uma "indústria do futuro"?

- Como a universidade pode apoiar a indústria? 
- Como aproximar a universidade da indústria?

- Como formar o profissional do futuro?

- Como o poder público deve participar da relação universidade-indústria?

O resultado desse debate, reforçado por publicações recentes, será a apresentado a seguir.

\section{Como é a indústria do futuro?}

Segundo os participantes do U4i World Café, a indústria será cada vez mais aberta, conectada e envolvida com a sociedade. O emprego das já mencionadas tecnologias da informação deve gerar novas formas de relacionamento entre empresas e clientes e também entre empresas de segmentos diversos. Essas novas conexões, por sua vez, podem gerar novas oportunidades de negócios.

A forma de produzir vai mudar. O barateamento de equipamentos de manufatura aditiva permitirá, por exemplo, que o produto, ou parte dele, seja produzido pelo próprio cliente em sua casa. Além disso, os tempos de ciclos de vida dos produtos devem continuar diminuindo, exigindo agilidade e redução de atividades sem agregação de valor em todos os setores da organização. Ao mesmo tempo, a sociedade deve cada vez mais valorizar empresas que conduzam seus negócios de forma sustentável, com especial atenção à minimização de impactos ambientais e à responsabilidade social.

Esse perfil, traçado pelos participantes do U4i World Café, coincide com a visão de especialistas. Os resultados do estudo Indústria 2027, encomendado pela Confederação Nacional da Indústria (CONFEDERAÇÃO NACIONAL DA INDÚSTRIA, 2018), apontam que, até o ano de 2027, todos os sistemas produtivos conviverão com tecnologias digitais avançadas, gerando modelos de negócio integrados, conectados e inteligentes. Essa forma de atuação permitirá a otimização de toda a cadeia produtiva através da combinação de escala com diferenciação e customização.

\section{O que falta para que a indústria nacional seja uma "indústria do futuro"?}

A baixa produtividade da indústria brasileira foi mencionada como uma das principais barreiras ao desenvolvimento da mesma. Essa baixa produtividade seria causada por um conjunto de fatores. A falta de mão de obra capacitada e a baixa eficiência do corpo técnico seriam alguns deles. O isolamento da indústria brasileira também foi mencionado como motivo da baixa 


\section{A universidade para a indústria do futuro}

produtividade e competitividade. Por ser muito focada no mercado nacional, a mesma não é pressionada a lançar produtos inovadores, com qualidade e preço disponíveis no exterior.

Segundo um dos participantes, não há cultura para inovar e empreender no Brasil. O brasileiro não se permite errar e, portanto, é avesso ao risco. O desenvolvimento dessa cultura precisaria ser incentivado através de programas de fomento governamentais e promovido nas universidades. Os empresários, por sua vez, precisam conscientizar-se de que devem assumir uma postura mais proativa e arrojada, dedicando-se cada vez mais à gestão estratégica de longo prazo de seus negócios.

Um panorama amplo do estado atual de desenvolvimento da indústria nacional é apresentado no relatório do estudo Indústria 2027 (CONFEDERAÇÃO NACIONAL DA INDÚSTRIA, 2018). É alarmante, por exemplo, que em 2017 apenas 2\% das empresas consultadas no estudo estejam no estágio mais avançado de digitalização, ou seja, são empresas integradas, conectadas e inteligentes. Aproximadamente 37\% das empresas ainda se encontram no estágio inicial de digitalização, correspondente à aplicação de tecnologias digitais apenas de forma isolada, ou seja, não-integrada.

\section{Como a universidade pode apoiar a indústria?}

Segundo os participantes, em primeiro lugar, é necessário melhorar a comunicação entre a universidade e a indústria. É necessário criar canais para que empresas possam, de forma centralizada, encaminhar suas demandas à universidade. Além disso, a universidade deve ampliar a divulgação das competências de seu corpo docente junto às empresas.

As empresas estariam abertas às mais diversas formas de cooperação, que não devem estar limitadas a projetos de pesquisa, desenvolvimento e inovação. Atividades de consultoria e capacitação devem ser ampliadas. Mencionou-se ser de grande valor a visão de um profissional externo à empresa no apoio à solução de problemas técnicos cotidianos.

O perfil dos cursos universitários brasileiros da área tecnológica também foi citado como entrave ao desenvolvimento industrial. Os cursos seriam excessivamente teóricos e, além disso, baseados em conhecimentos defasados. Muitos profissionais recém-formados precisariam de muito tempo para conseguir aplicar na prática o conhecimento teórico adquirido na universidade. Uma maior integração entre as empresas e as universidades foi citada como uma possível solução para esse problema. Essa integração permitiria que os estudantes tivessem maior contato com o ambiente profissional durante o curso e que os professores se mantivessem mais atualizados. 
De fato, a interação entre universidade e indústria é ainda bastante restrita no país. Segundo levantamento realizado por Lemos e Cario (2017), apenas 12,74\% dos grupos de pesquisa brasileiros cadastrados no $\mathrm{CNPq}$ declaram manter relacionamentos com a indústria. Em Santa Catarina, esse índice é apenas um pouco maior: 18,92\%. Como benefícios para professores e estudantes que se envolvem em projetos de cooperação universidade-indústria, esses mesmos pesquisadores apuraram a aquisição de conhecimento e habilidades mais próximas à realidade do mercado de trabalho, reforçando uma das observações dos participantes do U4i World Café.

\section{Como aproximar a universidade da indústria?}

Destacou-se que há pouca informação sobre as formas de iniciar um trabalho com a universidade. Para vencer esse problema, sugeriram-se a elaboração de cartilhas com procedimentos para as diversas formas de interação, como a captação e seleção de estagiários, a celebração de convênios de cooperação e a contratação de consultorias técnicas e cursos de curta duração.

Como estratégias para aproximar cada vez mais a universidade da indústria foram citados o desenvolvimento de pesquisas de pós-graduação diretamente nas empresas, a oferta de disciplinas optativas por profissionais do meio industrial e de disciplinas baseadas em projetos propostos por empresas. Também sugeriu-se a promoção de eventos universidade-empresa para apresentação de demandas e competências.

As sugestões apontadas pelos participantes encontram respaldo no trabalho publicado por Bruneel, D’Este e Salter (2010). Essa pesquisa avaliou as principais barreiras à cooperação entre universidade-indústria para desenvolvimento de projetos de pesquisa aplicada e inovação. Conflitos relacionados à forma de geração e disseminação do conhecimento foram apontados como sendo os principais desafios. Enquanto acadêmicos são medidos pela quantidade e qualidade dos resultados de pesquisa publicados, empresas precisam proteger e se apropriar do conhecimento gerado para desenvolver vantagem competitiva. Vencida essa barreira, ainda restam muitas vezes complexas negociações relacionadas à propriedade intelectual.

O estudo destaca o desenvolvimento de um sentimento de confiança interinstitucional como a principal forma de vencer esses obstáculos. A construção dessa confiança exige investimento de longo prazo em interações entre academia e indústria, visando à compreensão das diferenças de objetivos e formas de atuação (BRUNEEL; D’ESTE; SALTER, 2010).

Além disso, no Brasil, muitas vezes ainda é necessário vencer barreiras internas às universidades. De forma geral, ainda não há um entendimento mais amplo sobre o que é e quais 
os benefícios da cooperação universidade-indústria, gerando resistência interna e levando a discussões ideológicas (LEMOS; CARIO, 2017).

\section{Como formar o profissional do futuro?}

Segundo os participantes, há necessidade de formar profissionais com perfil interdisciplinar e que tenham condições de rapidamente aplicar conhecimento teórico na solução de problemas práticos. Como habilidade técnica básica para atuação na indústria do futuro foi mencionado o conhecimento de linguagens de programação, em especial no contexto da leitura e interpretação de sinais de sensores dos mais diversos tipos.

A importância de se formar pessoas preparadas para o novo paradigma industrial foi confirmada no estudo do Future of Jobs 2018, do Fórum Econômico Mundial. Segundo esse estudo, a maioria das empresas pesquisadas apontou a disponibilidade de pessoal capacitado como o fator mais importante na definição da localização geográfica de suas operações (WORLD ECONOMIC FORUM, 2018).

O estudo do WEF infere que, já em 2022, a introdução de novas tecnologias digitais demandará que profissionais concentrem-se no desenvolvimento de habilidades que não possam ser substituídas por máquinas, como a programação, o pensamento analítico e crítico e a criatividade (WORLD ECONOMIC FORUM, 2018).

\section{Como o poder público deve participar da relação universidade-indústria?}

O fomento a projetos de pesquisa, desenvolvimento e inovação e o estabelecimento de metas e políticas de longo prazo foram mencionadas como sendo as principais atribuições do poder público no contexto da cooperação entre universidade e indústria. Como formas específicas de fomento, foram sugeridas a concessão de bolsas para que alunos desenvolvam projetos nas empresas, o financiamento de projetos de cooperação que promovam o desenvolvimento de regiões mais atrasadas do estado e do país e o fomento à realização de estágios. Criticou-se a atual falta de continuidade dos programas de fomento. Essa falta de continuidade dificultaria a realização de projetos complexos, que demandem dedicação em longo prazo.

Criticou-se também a atual forma de atuação das universidades públicas. Essas seriam demasiadamente burocráticas e, por isso, muito lentas nas respostas às demandas da indústria. Além disso, apontou-se que os programas de pós-graduação deveriam atuar como vetores da 
interação universidade-indústria. Para tal, seria necessário que algumas políticas e legislações fossem revistas. Mencionou-se que as métricas utilizadas na avaliação de cursos de mestrado profissionais são praticamente as mesmas dos mestrados acadêmicos, ou seja, baseadas na quantidade e qualidade de publicações. Além disso, atualmente não seria permitido o acúmulo de bolsas de agências públicas de fomento com bolsas pagas por empresas. Essa situação dificultaria a captação de talentos para o desenvolvimento de projetos de pesquisa aplicada e inovação.

\section{CONSIDERAÇÕES FINAIS}

A produção industrial está diante de uma mudança de paradigma motivada pela integração de ferramentas avançadas de tecnologia da informação às máquinas e equipamentos e pela rápida ampliação da automatização de processos. Nesse contexto, espera-se que os centros tecnológicos das universidades assumam um papel de liderança, tanto no desenvolvimento e introdução de novas tecnologias, quanto na formação de profissionais alinhados a essa nova realidade. Para que as universidades possam atender a essa expectativa, é necessário que sejam mais ágeis e mais integradas ao meio industrial. Essa foi a uma das mensagens deixadas pelos participantes do U4i World Café.

Os resultados do U4i World Café serão um dos elementos utilizados para balizar o planejamento estratégico do Centro Tecnológico de Joinville (CTJ) da UFSC. O CTJ ambiciona ser um exemplo bem sucedido de integração entre indústria e universidade. O evento U4i é uma das ações nesse sentido. A iniciativa mais importante, no entanto, foi a transferência da sede da UFSC Joinville para o condomínio industrial Perini Business Park no início de 2018. O modelo de atuação resultante dessa mudança é inédito no país.

Da proximidade física entre a UFSC e as empresas do parque já resultaram diversas oportunidades de projetos e de estágios para alunos. Esses estudantes podem literalmente ir caminhando da universidade para a empresa e assim intercalar períodos de estudos com períodos de trabalho. Visitas técnicas realizadas no âmbito de disciplinas de graduação e pós-graduação também tornaram-se frequentes.

A mudança também motivou a criação do "Hub UFSC-Perini”. O Hub, criado e operacionalizado por um grupo formado por professores da UFSC e profissionais de empresas do parque, tem a função de facilitar a conexão de empresas com a universidade através, por exemplo, da organização de reuniões e visitas guiadas.

A UFSC também participa ativamente da concepção do Ágora Tech Park. O Ágora é um

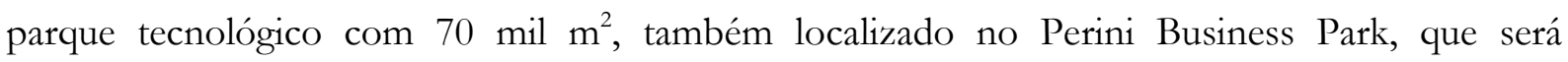


A universidade para a indústria do futuro

inaugurado em março de 2019. O Ágora será ocupado por diversos empreendimentos e iniciativas relacionadas à tecnologia e inovação, como incubadoras, aceleradoras, centros de pesquisa e startups.

$\mathrm{Na}$ área de ensino, a UFSC oferece em Joinville atualmente oito cursos de graduação e dois cursos de pós-graduação. Dentre os cursos de graduação está o Bacharelado Interdisciplinar, que está especialmente alinhado com as tendências apontadas durante o evento. Trata-se de um curso superior com apenas três anos de duração, com grade curricular flexível, que objetiva permitir que o aluno construa uma sólida base de conhecimento em ciências exatas e tecnologia de acordo com seus interesses e vocação.

\section{CRÉDITOS}

Comissão de organização do U4i 2018: Anne-Sophie Lohmeier (TH Ingolstadt), Leonardo Vinícius de Souza Silva (UFSC), Marcos Alves Rabelo (UFSC), Maurício de Campos Porath (UFSC), Modesto Hurtado Ferrer (UFSC), Xisto Lucas Travassos Júnior (UFSC).

Planejamento e condução do U4i World Café: Jonas Tilp (Treecom Coworking), Vanessa Aparecida Alves de Lima (UFSC), Xisto Lucas Travassos Júnior (UFSC).

Participantes do U4i World Café: Ademir Camillo Junior (SENAI), Alessandro Zimmer (TH Ingolstadt), Carlos Mauricio Sacchelli (UFSC), Carlos Henrique Barbado (BMW), Cristiano Vasconcellos Ferreira (UFSC), Dalton Hardt (4Factory), Edson Jeske (Gridya), Emerson Edel (Perini Business Park), Fábio Reway (TH Ingolstadt), Francielly Hedler Staudt (UFSC), Gabriel Bottós (Welle Laser), Gustavo Bay Gonçalves (Sensorville), Igor Luduwichack da Silva (Agrize), João Carlos Germani (Alutec), Jose Luiz Baccan (Siemens-Healthineers), Kleber Vieira de Paiva (UFSC), Leonardo Artur Biazi (SMETRO), Luís Fernando Peres Calil (UFSC), Maria Fernanda Monteiro (Pixida), Natanael Kaminski (Pollux), Ricardo Santin (BMW), Roberto Marques (Zeiss), Rodolfo Cereghino Lagreze (Embraco), Rodrigo Mendes (Rhode-Schwarz), Roger Cardoso dos Santos (Docol), Sueli Fischer Beckert (UFSC), Thiago Chiarotti de Oliveira (Schulz).

\section{REFERÊNCIAS}

BRUNEEL, Johan.; D'ESTE, Pablo; SALTER, Ammon. Investigating the Factors That

Diminish the Barriers to University - Industry Collaboration. Research Policy, v. 39, n. 7, 858868. 2010.

CONFEDERAÇÃO NACIONAL DA INDÚSTRIA. Indústria 2027: Estudo de sistema produtivo - Síntese dos resultados. Disponível em:

http://www.portaldaindustria.com.br/publicacoes/2018/5/industria-2027-estudo-de-sistemaprodutivo/ - industria-2027-estudo-de-sistema-produtivo-sintese-dos-resultados Acesso em 14 dez. 2018.

LEMOS, Dannyela da Cunha.; CARIO, Silvio Antônio Ferraz. University - Industry Interaction in Santa Catarina: Evolutionary Phases, Forms of Interaction, Benefits, and Barriers. RAI

Revista de Administração e Inovação, v. 14, n. 1., p. 16-29. 2017. 
A universidade para a indústria do futuro

WORLD ECONOMIC FORUM. The Future of Jobs Report 2018. Disponível em:

https://www.weforum.org/reports/the-future-of-jobs-report-2018 Acesso em 14 dez. 2018.

Recebido em: 06/03/2019

Aceito em: 08/08/2019 